\title{
The Schutz Formalism and the Kantowski-Sachs Quantum Cosmological Model
}

\author{
Andre Luiz Alves Lima* ${ }^{a b}$, Raphael Fracalossi ${ }^{a b}$, Flávio Gimenes Alvarenga ${ }^{a c}$ \\ ${ }^{a}$ Universidade Federal do Espírito Santo \\ ${ }^{b}$ Centro de Ciências Exatas \\ Departamento de Física \\ Av. Fernando Ferrari 514, Vitória, ES, Brasil, CEP: 29075-910 \\ ${ }^{c}$ Centro Universitário Norte do Espírito Santo \\ Departamento de Ciências Matemáticas e Naturais
}

Rodovia BR 101 Norte, Km 60, Bairro Litorâneo, São Mateus, ES, Brasil, CEP: 29932-540

E-mail: andrealves.fisegmail.com, rfracalossiegmail.com,

flavioalvarenga@ceunes.ufes.br

\begin{abstract}
In the scenario of quantizing gravity, quantum cosmology appears as an interesting application of any formalism which shows itself as a candidate of a fundamental theory. If one follows the standard model, the universe must have been, at some stage of its evolution, inside the limits of quite small scales, where quantum effects must have been significant. This quantum influence on the initial conditions of the universe might have important consequences on its actual stage. We present below the quantization of an anisotropic universe, the Kantowski-Sachs model, filled with a perfect fluid that is treated on the grounds of a formalism developed by B. Schutz in 1970. With this formalism, using the fluid as a clock, we find a parameter that "behaves like time", and thus may give the dynamics of the universe, otherwise frozen in an empty-universe model.
\end{abstract}

4th International Conference on Fundamental Interactions

August 1-7, 2010

Viosa, Brazil

\footnotetext{
*Speaker.
} 


\section{An anisotropic space-time.}

We will consider a model of an universe that is homogeneous but anisotropic [1]. Thus, we shall deal with a space-time such that the line element can be put in the form

$$
\mathrm{d} s^{2}=-N(t)^{2} \mathrm{~d} t^{2}+a(t)^{2} \mathrm{~d} r^{2}+b(t)^{2} \mathrm{~d} \Omega^{2},
$$

where $r$ is the radial polar coordinate, and $\mathrm{d} \Omega$ is the usual element of solid angle. This is known as the Kantowski-Sachs (KS) metric [2]. It is evident then that the space-time is passive of a slicing into spatial 3-surfaces of metric $g_{i j}$. The two different scale factors, $a(t)$ and $b(t)$, give the inhomogeneity, and with a suitable definition of these factors, the space-time can be identified with the FRW one [3]. So the FRW universe is a particular case of the KS universe.

We want to find a Lagrangian for this metric, so we we start with the Einstein-Hilbert action,

$$
s=\int \mathrm{d}^{4} x \sqrt{-g} \mathrm{R},
$$

where $g$ is the metric determinant, and $\mathrm{R}$ is the Ricci scalar. If one calculates the Ricci scalar for the metric given by (1.1) and put it into the action, one will find that

$$
s_{g}=\int \mathrm{d} t\left(2 N a-\frac{4 \dot{a} \dot{b} b}{N}-\frac{2 a \dot{b}^{2}}{N}\right)
$$

where we have neglected surface terms that would have zero variation, and we have neglected a global scale factor due the integration over the spatial part, that can be normalized. The integrand above is, thus, the Lagrangian for gravity, and we can make a suitable reparametrization, introducing a variable $c(t)$ given by $c(t)=a(t) b(t)$. With that, the gravitational Lagrangian takes the form

$$
\mathrm{L}_{g}=\frac{N a}{2}+\frac{\dot{a}^{2} c^{2}}{2 N a^{3}}-\frac{\dot{c}^{2}}{2 N a} .
$$

There are two canonical variables: the functions $a(t)$ and $c(t)$ that really are the scale factors (after the reparametrization); this is quite reasonable, since these are the functions that describe the liberty of the system. With this Lagrangian, one can find the conjugate momenta $p_{a}$ and $p_{c}$ and use those to find the Hamiltonian, that is given in the end by

$$
\mathrm{H}_{g}=\frac{N a^{3}}{2 c^{2}} p_{a}^{2}-\frac{N a}{2} p_{c}^{2}-\frac{N a}{2} .
$$

\section{Potentials of matter.}

Now, let us consider the dynamics of a perfect fluid composed of baryons with four-velocity $U_{v}$, in a space-time with metric coefficients given by $g_{\mu v}$. In 1970, Bernard Schutz developed a formalism to describe the four-velocity with the aid of six scalar potentials[4]1:

$$
U_{v}=\frac{1}{\mu}\left(\varphi_{, v}+\alpha \beta_{, v}+\theta S_{, v}\right) .
$$

\footnotetext{
${ }^{1}$ Such a construction is absolutely valid, though it uses more potentials to describe the fluid than the strictly necessary. That is done, nevertheless, since with six potentials it is possible to find evolution equations to each of them separately.
} 
Two of this potentials have a very familiar significance: in fact, $S$ is the entropy of the fluid, and $\mu$ its specific inertial mass, defined as $\mu=(\rho+p) / \rho_{0}$, in terms of the fluid density of rest mass $\rho_{0}$ and the density of total mas-energy $\rho$. But we have to be careful when talking about the rest-mass of a baryons fluid, since this rest-mass is not necessarily conserved. In order to be clear, then, we take the baryon number $N$, which is conserved, and we take the rest mass of hydrogen atom on its fundamental state, $m_{\mathrm{H}}$ to define the rest-mass of the fluid as $\rho_{0}=N m_{\mathrm{H}}$. With this, we define the fluids specific internal energy in terms of its total internal energy $\mathrm{E}$ as $\mathscr{E}=\mathrm{E} / N m_{\mathrm{H}}$. Then the density of total mass-energy can be written as $\rho=\rho_{0}(1+\mathscr{E})$. The other four potentials have a more indirect meaning, and $\alpha$ and $\beta$ are related to the circulation of the fluid.

We will suppose that we are in a co-moving frame, and the four-velocity is normalized, that is $U_{\mu} U^{v}=-1$, what, with (2.1), gives that $\mu$ is a function of all the other potentials:

$$
\mu^{2}=-g^{\sigma v}\left(\varphi_{, \sigma}+\alpha \beta_{, \sigma}+\theta S_{, \sigma}\right)\left(\varphi_{, v}+\alpha \beta_{, v}+\theta S_{, v}\right) .
$$

This can be a little bit simplified if we require that our perfect fluid presents no vortices, what is a good thing for the matter of an homogeneous universe. In this case, the $\alpha$ and $\beta$ potentials vanish, leaving us with

$$
\mu^{2}=-g^{\sigma v}\left(\varphi_{, \sigma}+\theta S_{, \sigma}\right)\left(\varphi_{, v}+\theta S_{, v}\right) .
$$

One can go a little further, and assume that the potentials are only functions of time, what gives finally $\mu^{2}=-g^{00}\left(\varphi_{, 0}+\theta S_{, 0}\right)^{2}$, or

$$
\mu^{2}=\frac{1}{N^{2}}(\dot{\varphi}+\theta \dot{S})^{2} .
$$

We will now find a formula for the pressure $p$ in terms of these potentials. The first law of thermodynamics (apllied per unit rest-mass) gives that

$$
\mathrm{d} \mathscr{E}+p \mathrm{~d}\left(1 / \rho_{0}\right)=T \mathrm{~d} S
$$

for the entropy $S$ and temperature $T$ of the fluid. Using the definition of $\mu$, one arrives at the result that

$$
\mathrm{d} p=\rho_{0} \mathrm{~d} \mu-\rho_{0} T \mathrm{~d} S .
$$

One can see with this relation that $p=p(\mu, S)$. We choose the equation of a perfect fluid, $p=\rho$, that gives

$$
(1+\mathscr{E}) \mathrm{d}\left[\ln (1+\mathscr{E})-\ln \rho_{0}\right]=T \mathrm{~d} S
$$

It follows that

$$
T=1+\mathscr{E}, \quad \text { and } \quad S=\ln (1+\mathscr{E})-\ln \rho_{0} .
$$

So $\rho_{0}$ can be written in terms of the entropy and the energy:

$$
\rho_{0}=(1+\mathscr{E}) e^{-S}
$$

Well, by the definitions given, $\mu=(\rho+p) / \rho_{0}=1+\mathscr{E}+p / \rho_{0}$, so the equation of state gives $(1+\mathscr{E})=\mu / 2$. Putting this into the last equation, one gets

$$
\rho_{0}=\frac{\mu}{2} e^{-S}
$$


Then we use the definition of $\rho$ and the equation of state to easily find

$$
p=\frac{\mu^{2}}{4} e^{-S} .
$$

Now, using the result of equation (2.2), one arrives finally at the desired formula:

$$
p=\frac{(\dot{\varphi}+\theta \dot{S})^{2}}{4 N^{2}} e^{-S}
$$

The action for the fluid is the usual, given by

$$
s=\int \mathrm{d}^{4} x \sqrt{-g} p .
$$

With the metric given in (1.1), we have that $\sqrt{-g}=N a b^{2} \sin \theta=N \sin \theta c^{2} / a$, so the fluid Lagrangian becomes, with the aid of (2.4) and neglecting a global factor due to the integration over space,

$$
\mathrm{L}_{f}=\frac{1}{4 N} \frac{c^{2}}{a}(\dot{\varphi}+\theta \dot{S})^{2} e^{-S}
$$

The Lagrangian gives the conjugate momenta $p_{i}=\partial L_{f} / \partial \dot{q}_{i}$, and one finds that

$$
p_{\varphi}=\frac{1}{2 N} \frac{c^{2}}{a}(\dot{\varphi}+\theta \dot{S}) e^{-S}, \quad p_{S}=\theta p_{\varphi}, \quad p_{\theta}=0
$$

With those, it is possible to construct the Hamiltonian for the fluid, that is given by

$$
\mathrm{H}_{f}=\frac{N a}{c^{2}} e^{S} p_{\varphi}^{2}
$$

\section{Canonical transformation and a reordering.}

The total Hamiltonian is the sum of the gravity and the fluid ones, that is, the sum of (1.2) and (2.5):

$$
\mathrm{H}=\frac{N a}{2 c^{2}}\left(a^{2} p_{a}^{2}-c^{2} p_{c}^{2}-c^{2}+2 e^{S} p_{\varphi}^{2}\right) .
$$

The trick that makes the Schutz formalism attractive relies in the following: lets return to the fluids hamiltonian, (2.5), which had as canonical variables the entropy, $S$, and the potential $\varphi$, and make a canonical transformation given by

$$
t=-p_{S} e^{-S} p_{\varphi}^{-2}, \quad p_{t}=p_{\varphi}^{2} e^{S} ; \quad \bar{\varphi}=\varphi-2 \frac{p_{S}}{p_{\varphi}}, \quad p_{\bar{\varphi}}=p_{\varphi}
$$

This transformation directly linearises the fluids hamiltonian, which becomes

$$
\mathrm{H}_{f}=\frac{N a}{c^{2}} p_{t} .
$$

We can put that into the total Hamiltonian now, and get

$$
\mathrm{H}=\frac{N a}{2 c^{2}}\left(a^{2} p_{a}^{2}-c^{2} p_{c}^{2}-c^{2}+2 p_{t}\right) .
$$


This Hamiltonian is linear in the new canonical momenta $p_{t}$. So, when we use the canonical quantization procedure due to Dirac, of putting hats on top of the canonical momenta and transforming them into derivatives, as $p_{\xi} \rightarrow \hat{p}_{\xi}=-i \partial_{\xi}$, we get a Hamiltonian operator

$$
\hat{\mathrm{H}}=\frac{N a}{2 c^{2}}\left(-a^{2} \frac{\partial^{2}}{\partial a^{2}}+c^{2} \frac{\partial^{2}}{\partial c^{2}}-c^{2}+2 i \frac{\partial}{\partial t}\right)
$$

that has a first order derivative on the parameter $t$. Applying this as the annihilator of the wave function of the universe, $\Psi(a, c, t)$, we get the Wheeler-DeWitt equation. With a reparametrization of the variable $t$ as $t \rightarrow 2 t$, that reads

$$
\left\{-a^{2} \frac{\partial^{2}}{\partial a^{2}}+c^{2} \frac{\partial^{2}}{\partial c^{2}}-c^{2}+i \frac{\partial}{\partial t}\right\} \Psi(a, c, t)=0 .
$$

One has to be careful, though, when using this process of quantization, about the matter of ordering. For, when the canonical variables are transformed into operators, these operators have no longer the need to be commutative and thus the order in which they are written becomes highly relevant. The most logical way of writing the operator is the one that makes it symmetric, since we want it to be an observable. That is, given two functions sufficiently well behaved $\zeta$ and $v$, we want to have $(\hat{\mathrm{H}} \zeta, v)=(\zeta, \hat{\mathrm{H}} v)$.

To achieve that, we make the following reordering:

$$
x^{2} \hat{p}_{x}^{2} \rightarrow \frac{1}{2}\left\{\left[\hat{p}_{x}\left(x^{2}\right)\right] \hat{p}_{x}+x^{2} \hat{p}_{x}^{2}\right\},
$$

which gives, after a reparametrization to absorb the constant factor, the following correspondence:

$$
-a^{2} \hat{p}_{a}^{2} \rightarrow a^{2} \partial_{a}^{2}+2 a \partial_{a} ; \quad-c^{2} \hat{p}_{c}^{2} \rightarrow c^{2} \partial_{c}^{2}+2 c \partial_{c} .
$$

This makes $\hat{\mathrm{H}}$ symmetric, and is the appropriate ordering. The final Wheeler-DeWitt equation is, thus,

$$
\left\{-a^{2} \frac{\partial^{2}}{\partial a^{2}}-2 a \frac{\partial}{\partial a}+c^{2} \frac{\partial^{2}}{\partial c^{2}}+2 c \frac{\partial}{\partial c}-c^{2}+i \frac{\partial}{\partial t}\right\} \Psi(a, c, t)=0 .
$$

\section{Conclusion.}

The Wheeler-DeWitt equation (3.2) is a Schrödinger-like equation, with the linear variable $t$ playing the role of time. One can think formally of a "stationary wave function" $\Psi(a, c, t)=$ $\psi(a, c) e^{-i E t}$, where $E$ is a constant. If the function $\psi$ can be written as $\psi(a, c)=\Upsilon(a) \Phi(c)$ we find the two following differential equations, where a dot denotes derivation with respect to $a$, and a prime denotes differentiation with respect to $c$ :

$$
a^{2} \ddot{\Upsilon}+2 a \dot{\Upsilon}+(E-\sigma) \Upsilon=0
$$

which is an Euler equation, with $\sigma$ as a constant of separation, and

$$
c^{2} \Phi^{\prime \prime}+2 c \Phi^{\prime}-\left(c^{2}+\sigma\right) \Phi=0
$$


which is a Bessel equation. The formal solution of the universe wave function is, thus,

$$
\Psi(a, c, t)=\frac{1}{\sqrt{c}}\left(A_{1} K_{v}(c)+A_{2} I_{v}(c)\right)\left(B_{1} a^{n_{+}}+B_{2} a^{n_{-}}\right) e^{-i E t}
$$

with $v=\sqrt{\sigma+1 / 4}$ and $n_{ \pm}=-1 / 2 \pm \sqrt{1 / 4-(E-\sigma)}$. One should still apply boundary conditions to this function. For example, it must go to zero as $c \rightarrow \infty$, so the modified Bessel functions of the first kind cannot be present in the solution. When $c$ goes to zero, the function is singular, but it does not diverge. Actually, since the Bessel functions of the second kind fall off exponentially for $c \rightarrow 0$, what is found is that $\Psi \rightarrow 0$ as $c \rightarrow 0$. At last, one must construct a wave packet over all the energies, weighting with some suitable function.

So we have found a Schrödinger-like equation for the wave function of the universe introducing a perfect fluid and using one of its canonical momenta as the time variable. Is this coherent? One can argue that it makes no sense to identify a temporal parameter in this case, since the theory plays its role over the space-like 3-surfaces of space-time, that are taken, each, in different instants of time, what means that the time is frozen. Henceforth, the variable $t$ found in the canonical transformation (3.1) cannot possibly be the time. We say that this is not such a definitive answer. For, when the theory is constructed, an infinite quantity of degrees of freedom are frozen, and only a few are considered. This means that among the infinite degrees of freedom frozen, there may be some, even lying on the 3 -surfaces, which are responsible for the intrinsic time evolution. The theory then unfreezes some of these degrees of freedom, giving back the time. And, indeed, if one looks carefully, the "unfrozen" degree of freedom here, responsible for the linear momentum in the Hamiltonian, is not a random variable. If we look to the canonical transformations (3.1), we will see to whom the "time" parameter is connected: $t=-p_{S} e^{-S} p_{\varphi}^{-2}-$ The new "time" is constructed, remarkably, from nothing less than the entropy of the fluid.

\section{References}

[1] C. Simeone, Global Phase Time and Wave Function for the Kantowski-Sachs Anisotropic Universe, Gen.Rel.Grav. 34, 1887-1893 (2002) [gr-qc/ $0208086 \mathrm{v1}$ ]

[2] R. Kantowski; R.K. Sachs, Some Spatially Homogeneous Anisotropic Relativistic Cosmological Models, J. Math. Phys. 7, 443 (1966)

[3] H. Conradi, Quantum Cosmology of Kantowski-Sachs like Models, Class.Quant.Grav. 12 (1995) 2423-2440 [gr-qc/9412049v3]

[4] B. F. Schutz, Perfect Fluids in General Relativity: Velocity Potentials and a Variational Principle, Phys. Rev. D 2, 2762-2773 (1970) 